

\title{
Towards a 3D dynamo model of the PMS star BP Tau
} Nicolas Bessolaz, A.S. Brun

\section{To cite this version:}

Nicolas Bessolaz, A.S. Brun. Towards a 3D dynamo model of the PMS star BP Tau. Astronomical Notes / Astronomische Nachrichten, 2011, 332 (9-10), pp.1045-1051. 10.1002/asna.201111612 . cea00828173

\section{HAL Id: cea-00828173 https://hal-cea.archives-ouvertes.fr/cea-00828173}

Submitted on 24 Sep 2019

HAL is a multi-disciplinary open access archive for the deposit and dissemination of scientific research documents, whether they are published or not. The documents may come from teaching and research institutions in France or abroad, or from public or private research centers.
L'archive ouverte pluridisciplinaire HAL, est destinée au dépôt et à la diffusion de documents scientifiques de niveau recherche, publiés ou non, émanant des établissements d'enseignement et de recherche français ou étrangers, des laboratoires publics ou privés. 


\title{
Towards a 3D dynamo model of the PMS star BP Tau
}

\author{
N. Bessolaz ${ }^{\star}$ and A.S. Brun \\ Laboratoire AIM Paris-Saclay, CEA/Irfu Université Paris-Diderot CNRS/INSU, 91191 Gif-sur-Yvette Cedex, France
}

Received 2011 Oct 4, accepted 2011 Oct 28

Published online 2011 Dec 12

Key words convection - magnetohydrodynamics (MHD) - stars: individual (BP Tau) - stars: pre-main sequence - stars: rotation - T Tauri stars - turbulence

Studying how convective and magnetic properties of pre-main sequence stars change during their evolution towards the zero-age main sequence is a growing area of research triggered by the development of efficient spectropolarimeters. 3D simulations can help to identify the key parameters to understand the diversity (strength, topology) of magnetic fields observed. We present results of a dynamo computation done with the ASH code for a $0.7 \mathrm{M} \odot$ pre-main sequence star with a 7.6 day rotation period which is nearly fully convective, using a realistic stratification contrast to resolve $90 \%$ of the convective zone. This star corresponds to the target star BP Tau already observed with spectropolarimetry (Donati et al. 2008). We particularly compare the magnetic field properties found in our simulation with the observational constraints.

(c) 2011 WILEY-VCH Verlag GmbH \& Co. KGaA, Weinheim

\section{Introduction}

Exploring how the magnetic properties of pre-main sequence stars are influenced by the change of their internal structure during evolution is very interesting as it can potentially improve our current understanding of stellar formation and evolution in the perspective of building more self-consistent theoretical models including magnetic field. The progress made over the last decade with spectropolarimetric observations using high resolution NIR instruments such as Narval and Espadons, seem to emphasize that the appearance and the modification of the size of the radiative core in a star modifies strongly the large scale magnetic field strength and topology. Fully convective stars both in the T Tauri phase such as BP Tau and AA Tau (Donati et al. 2008, 2010) and in mid M dwarfs during the main-sequence phase (Morin et al. 2008) present strong magnetic fields with mainly large-scale field dominated by dipolar and octupolar components. By contrast, pre-main sequence stars such as V2129 Oph where a radiative zone has already developed present weaker dipole field component with a much more complex field at smaller scale (Donati et al. 2007 and see also the paper of Scott Gregory in this volume).

The possibility to reproduce the topology and magnitude of such magnetic fields from first principles by carrying out fully 3D MHD simulations including a realistic stratification within stars is a very challenging goal. It will further helps us predict their evolution and general properties such as their rotation. Indeed, there are many evidences that magnetic fields play an important role to extract angular momentum by star-disc-wind interactions particularly during the pre-main sequence phase (Bessolaz et al. 2008, Bouvier 2009). Besides, star-disc interactions involving Clas-

\footnotetext{
^ Corresponding author: Nicolas.Bessolaz@cea.fr
}

sical T Tauri stars and the resulting stellar spin evolution depend strongly on the stellar magnetic field strength and topology. Thus, understanding the generation of the large scale magnetic field by dynamo action, arising from interplay between rotation and convection in the stellar interiors, is of fundamental interest. Bessolaz \& Brun (2011) have already shown how properties of giant cells in deep stellar convective zone and their associated mean flows such as differential rotation depend strongly on the depth of convective zones. Goudard \& Dormy (2008) in the Boussinesq approximation showed, near the onset of the dynamo threshold, that we can have transition from steady dipolar dominated dynamo to much complex smaller-scale dynamo with quasy-periodic reversal of the magnetic field when the depth of the convection zone becomes smaller than $0.4 R_{*}$. In the anelastic approximation, fully convective simulations for main-sequence M dwarfs carried out by Browning (2008) show significant large-scale magnetic field. But the stratification is very different in pre-main sequence stars since they are still in gravitational contraction and nuclear reactions have not yet started. Further, these young stars rotate much faster than the Sun which implies very different magnetic properties as demonstrated by Brown et al. $(2010,2011)$ for models of young suns using solar stratification.

In this paper, we present an attempt to model the dynamo action of a $0.7 \mathrm{M}_{\odot}$ pre-main sequence star with a 7.6 days rotation period which is nearly fully convective, using a realistic stratification contrast to resolve $90 \%$ of the convective zone. We first present in Sect. 2 the setup of the hydrodynamic progenitor and the resulting convective properties in such a star. Next, we study in Sect. 3 the magnetic properties resulting from a dynamo action and particularly the large scale magnetic field. Finally, we draw conclusions in Sect. 4. 

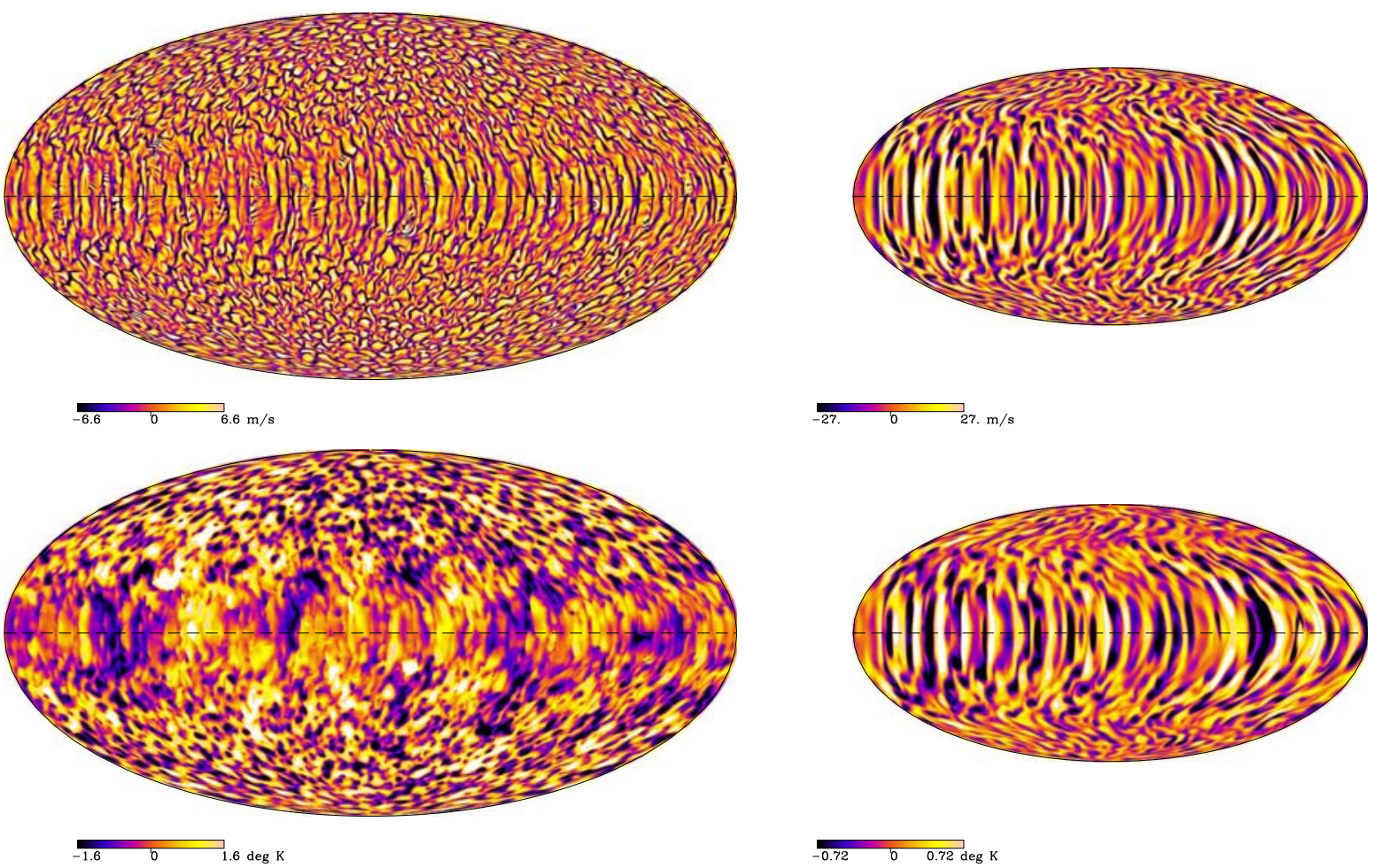

Fig. 1 (online colour at: www.an-journal.org) Convective structures (top) and temperature fluctuations (bottom) within the star at different depths: $r=0.97 R_{*}$ (left) and $r=0.65 R_{*}$ (right). All are shown, using Mollweide projection, with dark/blue tones indicating inward/negative flows and temperature fluctuations, and bright orange/yellow tones upward/positive ones.



Fig. 2 Local density scale height (solid line), density (dashed line) and temperature (dash-dotted line) radial distributions of our BP Tau model.

\section{Numerical setup}

The Anelastic Spherical Harmonic (ASH) code used for our computation solves the full set of 3-D MHD anelastic equations of motion (see Brun et al. 2004 for equations) in a rotating spherical shell on massively-parallel computer architectures.

The 1D stellar model for BP Tau is computed from the stellar evolution code CESAM (Morel 1997) as described in Bessolaz \& Brun (2011). Such a star is fully convective until it reaches 3.76 Myr. We choose the initial condition for our model just after the appearance of the radiative core at $4 \mathrm{Myr}$ in order to avoid the central singularity not yet resolved by the ASH code. At this time, the stellar radius is $1.224 \mathrm{R}_{\odot}$, the base of the convection zone is at $0.18 \mathrm{R}_{\odot}$ and the stellar luminosity is $0.4 \mathrm{~L}_{\odot}$. Moreover, the mass stratification (see Fig. 2) within the star is really different from the Sun since $87 \%$ (vs. $2.8 \%$ ) of the mass is localized within the convective zone and the central density is only $3.29 \mathrm{~g} \mathrm{~cm}^{-3}$ (vs. $160 \mathrm{~g} \mathrm{~cm}^{-3}$ ). The outer edge is at $r=0.97 R_{*}$ with a density scale height $H_{\rho}=14 \mathrm{Mm}$ giving a radial density contrast of 360 . The inner tangent cylinder (i.e. the imaginary cylinder aligned with the rotation axis at the bottom of the domain) cuts through the stellar surface at the latitude $81.6^{\circ}$, thus allowing efficient connectivity between low and high latitude regions.

Our grid resolution is $\left(N_{r}, N_{\theta}, N_{\phi}\right)=(512,1024,2048)$ with top values for the viscous $(\nu)$ and thermal $(\kappa)$ diffusivities coefficients of $1.1 \times 10^{12} \mathrm{~cm}^{2} \mathrm{~s}^{-1}$, corresponding to a Prandtl number of unity. The hydrodynamical progenitor with a rotation period of 7.6 days is computed during 10000 days reaching a turbulent convective statistically steady state in the last 1000 days.

\subsection{Convection properties}

The Rayleigh ( $\mathrm{Ra}$ ), Taylor (Ta), convective Rossby $\left(\mathrm{Ro}_{\mathrm{c}}\right)$, and Reynolds ( $\mathrm{Re}$ ) dimensionless numbers defined as in Bessolaz \& Brun (2011) at mid-depth are respectively equal to $\mathrm{Ra}=3.6 \times 10^{9}, \mathrm{Ta}=1.8 \times 10^{10}, \mathrm{Ro}_{\mathrm{c}}=0.14$, and $\mathrm{Re}=$ 


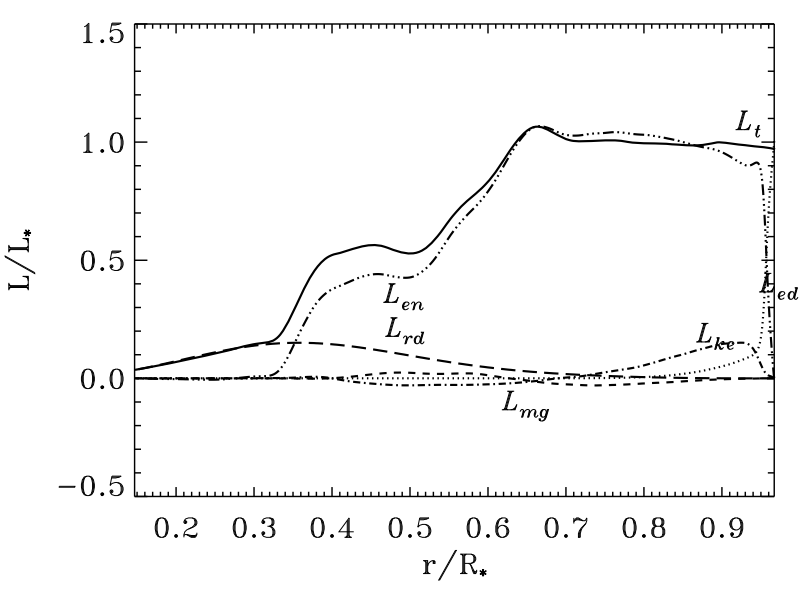

Fig.3 (online colour at: www.an-journal.org) Temporal average of radial energy flux balance converted and normalized to stellar luminosity. The energy mainly released from gravitational contraction is transported by convection $\left(L_{\mathrm{en}}\right)$, by radiation $\left(L_{\mathrm{rd}}\right)$, by kinetic energy $\left(L_{\mathrm{ke}}\right)$, by Poynting flux $\left(L_{\mathrm{mg}}\right)$ and by the unresolved eddy flux $\left(L_{\mathrm{ed}}\right)$ near the surface.

1100 which shows a highly turbulent state with a strong influence of rotation on convection.

For this model, rotating at a 3.7 times solar rate, we observe clearly the reminiscence of "banana cells" at low latitudes oriented in the North/South direction close to the stellar surface (see Fig. 1) which was non visible at solar rotation rate in the same kind of models presented in Bessolaz \& Brun (2011). However, the very low density scale height close to the stellar surface $\left(H_{\rho}=14 \mathrm{Mm}\right.$ at $r=0.97 R_{*}$ ) chosen for this model provides much more complex convective structures than previous models of fast rotators like in Brown et al. (2008). We can observe the asymmetry between broad upflows and narrow faster downflows due to stratification. At high latitudes, convective cells have smaller scales and are more isotropic but the transition is not linked to the position of the tangent cylinder which is very close to the axis but instead to the fact that convection is not really effective in this star below $0.4 R_{*}$, probably due to two effects; first most of the energy is carried by radiation and flows are weak below this depth (see Fig. 3) and second a strong shear flow is visible in the differential rotation profile at this location in our model (see Fig. 4), somewhat inhibiting convective motion at this depth. The longitudinal extent of the convective cells are also reduced, e.g. the dominant azimuthal $\mathrm{m}$ wavenumber is higher, as predicted by theory for fast rotators (e.g. Glatzmaier \& Gilman 1982). Even at this high rotation rate, we do not observe active nest of convection as in Brown et al. (2008). This is likely due to our choice of parameter with $\operatorname{Pr}=1$. The radial convective velocity profile peaks at $r=0.85 R_{*}$ around $46 \mathrm{~m} \mathrm{~s}^{-1}$ but reaches $5-10 \mathrm{~m} \mathrm{~s}^{-1}$ in the bulk of the convective zone which is a consequence of both the weak stellar luminosity of $0.4 \mathrm{~L}_{\odot}$ and the smaller stratification with respect to the Sun. By looking at the temperatures fluctuations maps, we can see the strong correlations between radial velocities and
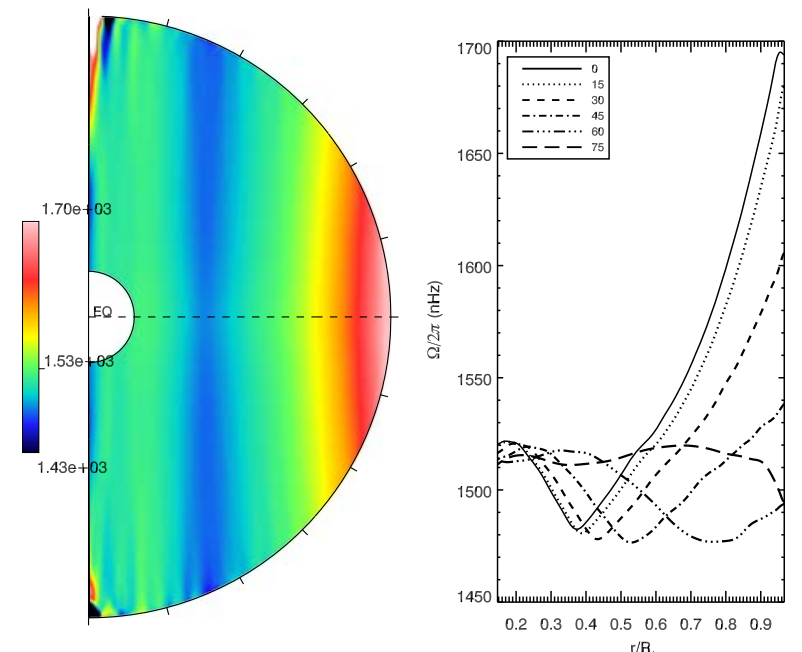

Fig. 4 Temporal and azimuthal averages of angular velocity profile in the meridional plane (left) and radial cuts for specific latitudes (right).

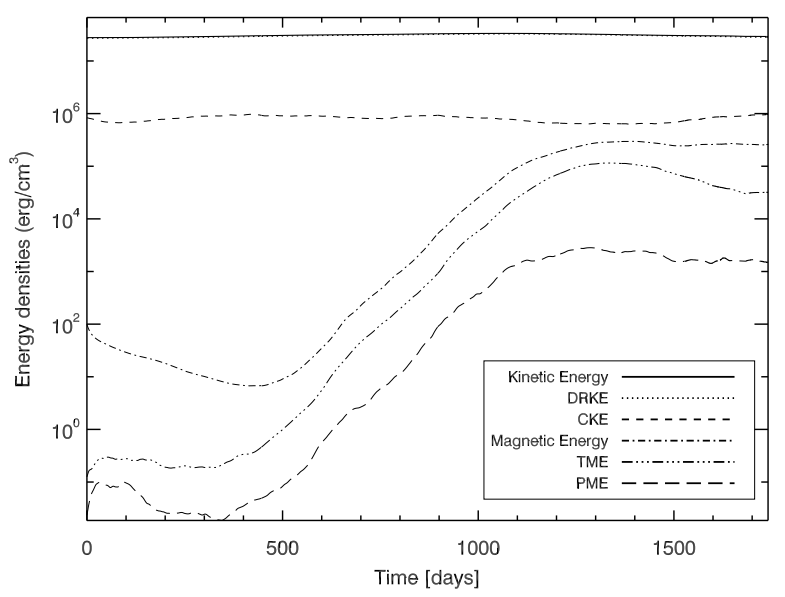

Fig. 5 Temporal evolution of the volume-integrated total kinetic energy (KE), kinetic energy in differential rotation (DRKE), total magnetic energy (ME), magnetic energy in the axisymmetric toroidal component (TME) and poloidal component (PME).

temperatures which transport efficiently heat outwards, especially in the downflow lanes which are associated with intense cool spots. We can notice the spatial coherence of convective cells with depth, as the strongest downflows cross most of the domain.

Figure 3 shows how the energy is transferred radially on average within the star. First, only $3 \%$ of the stellar luminosity is reached at the inner edge pointing out that the main source of energy in this pre-main sequence star is provided by the gravitational contraction which is included in the equations as described in Bessolaz \& Brun (2011) by adding an energy source term operating through the whole convective zone and proportional to temperature (Iben 1965). The stellar luminosity is reached near $r=0.7 R_{*}$. Convection is efficiently transporting the heat outwards only in the outer $60 \%$ of the envelope. The transport of energy by viscosity 

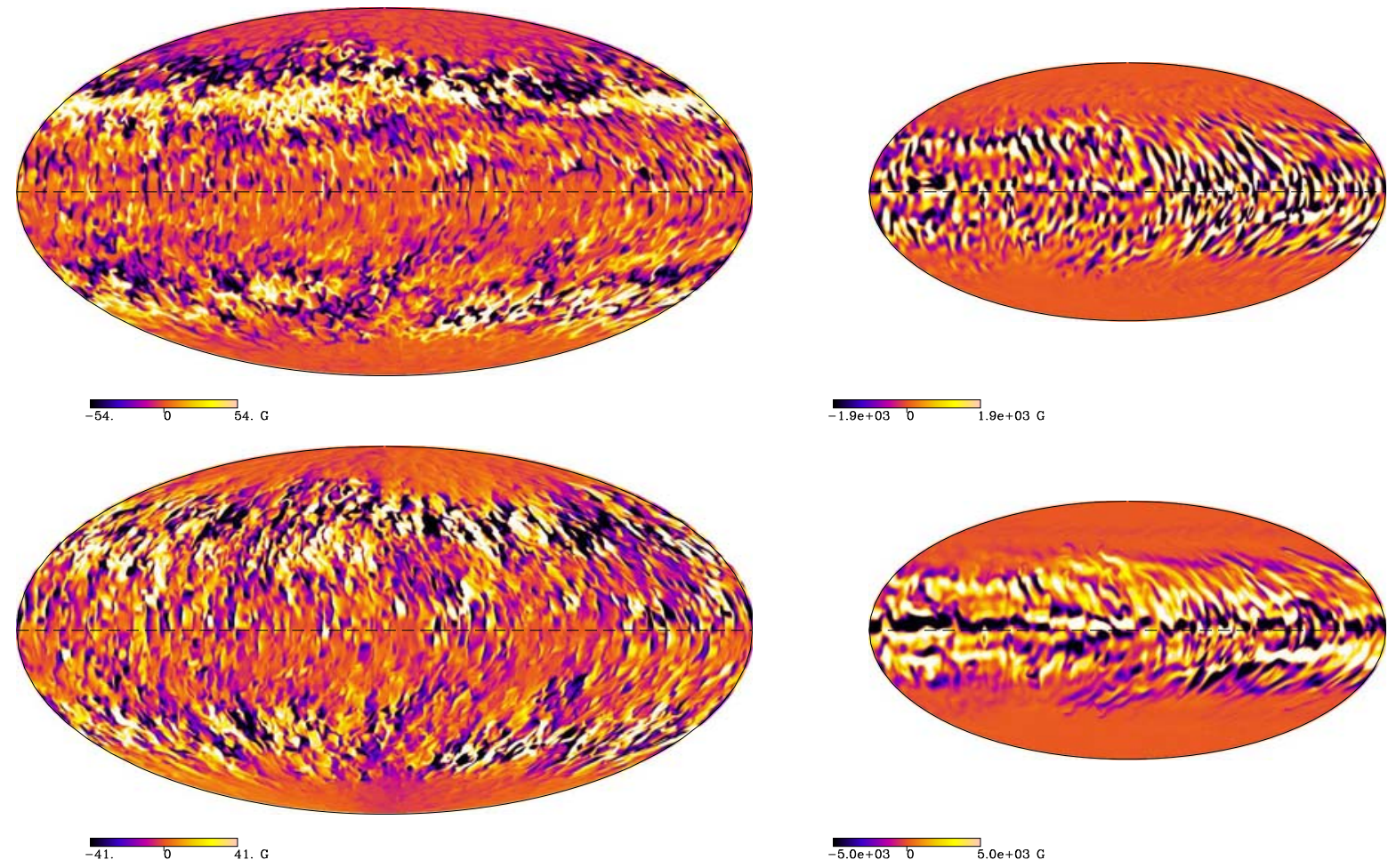

Fig. 6 (online colour at: www.an-journal.org) Radial magnetic field (top) and toroidal magnetic field (bottom) within the star at different depths: $r=0.97 R_{*}$ (left) and $r=0.65 R_{*}$ (right). All are shown, using Mollweide projection, with dark/blue tones indicating negative polarity and bright orange/yellow tones positive polarity.

(dashed line) is negligible in this model as well as the transport by the unresolved eddy flux. For the latter, its contribution to match the superadiabatic entropy gradient dominates only in the outer $5 \%$ of the star. We also can notice that the transport of energy by the radial Poynting flux is very weak.

The differential rotation is dominated by band of slow/fast jets. It is mainly cylindrical as in Ballot et al. (2007) and Brown et al. (2008). This is in agreement with Taylor-Proudman constraint that faster rotating flows are quasi $2 \mathrm{D}$ and aligned with the rotation axis. The differential rotation profile (see Fig. 4) is prograde with a relative contrast of $13 \%$ near the surface between equator and high latitudes $\left(75^{\circ}\right)$. This differential rotation profile is kept almost unchanged during our dynamo simulation. However, observations do not report a significant differential rotation (Donati et al. 2008) which may be coherent with the fact that the magnetic field on our dynamo simulation is too weak to modify the angular momentum balance. Mean field models of slow rotating $\mathrm{M}$ dwarfs on the main sequence computed by Küker \& Rüdiger (2005) also exhibit a weak differential rotation with a contrast up to only $2 \%$.

\section{Onset of dynamo and internal transport processes}

We choose the magnetic diffusivity in order to have a magnetic Prandlt number equal to one. The boundary condition for the magnetic field is perfect conductor at the inner edge and potential at the outer edge. Then, we initialize the magnetic computation with a multipole $l=3, m=2$ seed field where the mean magnetic energy is only $10^{-5}$ of the total kinetic energy. After an initial transient phase where the magnetic energy decreases by one order of magnitude, we observe its growth during 500 days in Fig. 5 due to dynamo action. The saturation of the dynamo is reached in the last 400 days. The magnetic Reynolds number based on rms velocities at mid-depth is $\mathrm{Rm}=1000$ but reaches 2500 near the surface. At this stage, the magnetic energy represents only $1 \%$ of the total kinetic energy and $27 \%$ of the convective kinetic energy as shown in Fig. 5.

\subsection{Magnetic properties}

The axisymmetric poloidal magnetic field component (PME) contributes only for $1 \%$ to the total magnetic energy whereas the toroidal magnetic component (TME) represents a large contribution between $12.5 \%$ (at $t=1700 \mathrm{~d}$ ) and $44.5 \%$ (at $t=1400 \mathrm{~d}$; see Fig. 5) without the need of a tachocline in this nearly fully convective star. This is most likely due to an efficient $\omega$-effect within the convective zone as seen in Brown et al. (2010). Thus, the fluctuating magnetic field components vary between $54 \%$ and $87 \%$ which is an intermediate behaviour between Brun et al. (2004) and Brown et al. (2010) which study thinner convective zones respectively at solar and faster rotation rates. 




Fig. 7 Magnetic energy components as function of depth. Total magnetic energy (ME), axisymmetric poloidal (PME), toroidal (TME) and fluctuating magnetic fields (FME) are shown.

In Fig. 6, we observe a global organization of magnetic fields with two magnetic wreathes at mid-latitudes which are very turbulent. However, they do not span the whole convective zone like in young main-sequence solartype stars (Brown et al. 2010) and they are concentrated near the surface for $r>0.85 R_{*}$. The toroidal magnetic field strength reaches $9 \mathrm{kG}$ and the poloidal one reaches $4.7 \mathrm{kG}$ at $r=0.85 R_{*}$. A striking feature is the very weak activity at high latitude which is coherent with the fact that the dynamo action is very weak in the $40 \%$ most inner region of the star (see Fig. 7) and mainly concentrated between $0.4 R_{*}$ and $0.8 R_{*}$. However, the strength of magnetic fields for $r>0.25 R_{*}$ is always larger than the seed field introduced at the initialization stage of our dynamo run although it is hard to disentangle if it is the consequence of an inward diffusion of the field from a larger radius or instead a weak local generation of magnetic field.

We can also notice the imprints of convective patterns on magnetic fields at low latitudes with concentration of fields in the downflow plumes. The radial magnetic field component reaches $54 \mathrm{G}$ near the surface with maximal magnitude around $1000 \mathrm{G}$. The toroidal magnetic field component reaches $41 \mathrm{G}$ near the surface with maximal magnitude around $400 \mathrm{G}$. A magnetic field reconstruction both within the star and its atmosphere by performing potential extrapolation is presented in Fig. 8. We show that the topology of the axisymmetric magnetic field evolves very much during the 400 days after reaching the saturation state both at large scales and small scales. For instance, there is a clear change of polarity in the southern hemisphere 282 days after the beginning of the saturation stage. We also find a trend towards a global change of polarity in the non axisymmetric part of the magnetic field by keeping only the first modes in our spherical harmonics analysis. For instance, the dipolar component is firstly dominated by the $m=1$ equatorial mode before becoming mostly axisymmetric which is a clue of a potential magnetic cycle in our model as shown in Brown et al. (2011). We intend to run this model even longer to make this change of polarity more obvious.

\subsection{Comparison of the surface magnetic field with observations}

Donati et al. (2008) reported a dipolar component of $1.2 \mathrm{kG}$ and an octupolar component of $1.6 \mathrm{kG}$ for BP Tau with strength in the radial and toroidal magnetic fields of $2 \mathrm{kG}$ and few $100 \mathrm{G}$ respectively. In Fig. 9, we perform a spherical harmonics analysis keeping modes up to $l=10$ to have the same spatial resolution as the one used in observational data processing. We apply this spherical harmonic filter near the current end of our simulation (i.e. at $t=1700 \mathrm{~d}$ ). We compare our reconstructed magnetic field with the observations in Fig. 9. Although we can find some qualitative agreement in the spatial distribution of the magnetic field except at the pole as already discussed previously, the magnitude of the radial and toroidal components found in our simulations is at least one order of magnitude weaker with maximal values around $50 \mathrm{G}$ and $26 \mathrm{G}$ respectively.

Besides, we find that the dipolar component is only $3 \mathrm{G}$ and that the octupolar component reaches $10 \mathrm{G}$. However, this weak large scale magnetic field is consistent with the fact we do not see a change in the differential rotation during our dynamo simulation with respect to the hydrodynamic simulation progenitor studied in Sect. 2, even if the Maxwell stress is far from being negligible in the transport of angular momentum but is counterbalanced by an efficient meridional circulation.

By looking at spectra of the magnetic energy density, we find that close to the stellar surface at $r=0.97 R_{*}$, the magnetic field energy is dominated by modes $10<l<50$ but at larger depth up to $r=0.65 R_{*}$, the spectra is much more flat with significant magnetic energy for $l<5$. For instance, the radial magnetic field component is $230 \mathrm{G}$ keeping modes up to $l=5$, the toroidal one reaches $1800 \mathrm{G}$ and the dipolar component reaches $15 \mathrm{G}$.

\section{Conclusion}

The first 3D simulation of a nearly fully convective PMS star using a realistic stratification has been carried out to compare with the observed target star BP Tau. We get self-consistently convection, rotation, meridional circulation flows and dynamo action with the production of a large scale magnetic field. The amplitude of this large scale magnetic field is weak and not consistent with recent spectropolarimetric measurements of BP Tau. However, our dynamo model undergoes a polarity change which is a clue of a potential magnetic cycle as it seems to be reported observationally for the very similar CTTS AA Tau (Donati et al. 2010) and theoretically by Brown et al. (2011) for fast rotators of solar-like stars on the main sequence. This simulation needs to be computed on longer time scale to conclude about its global magnetic field properties. 


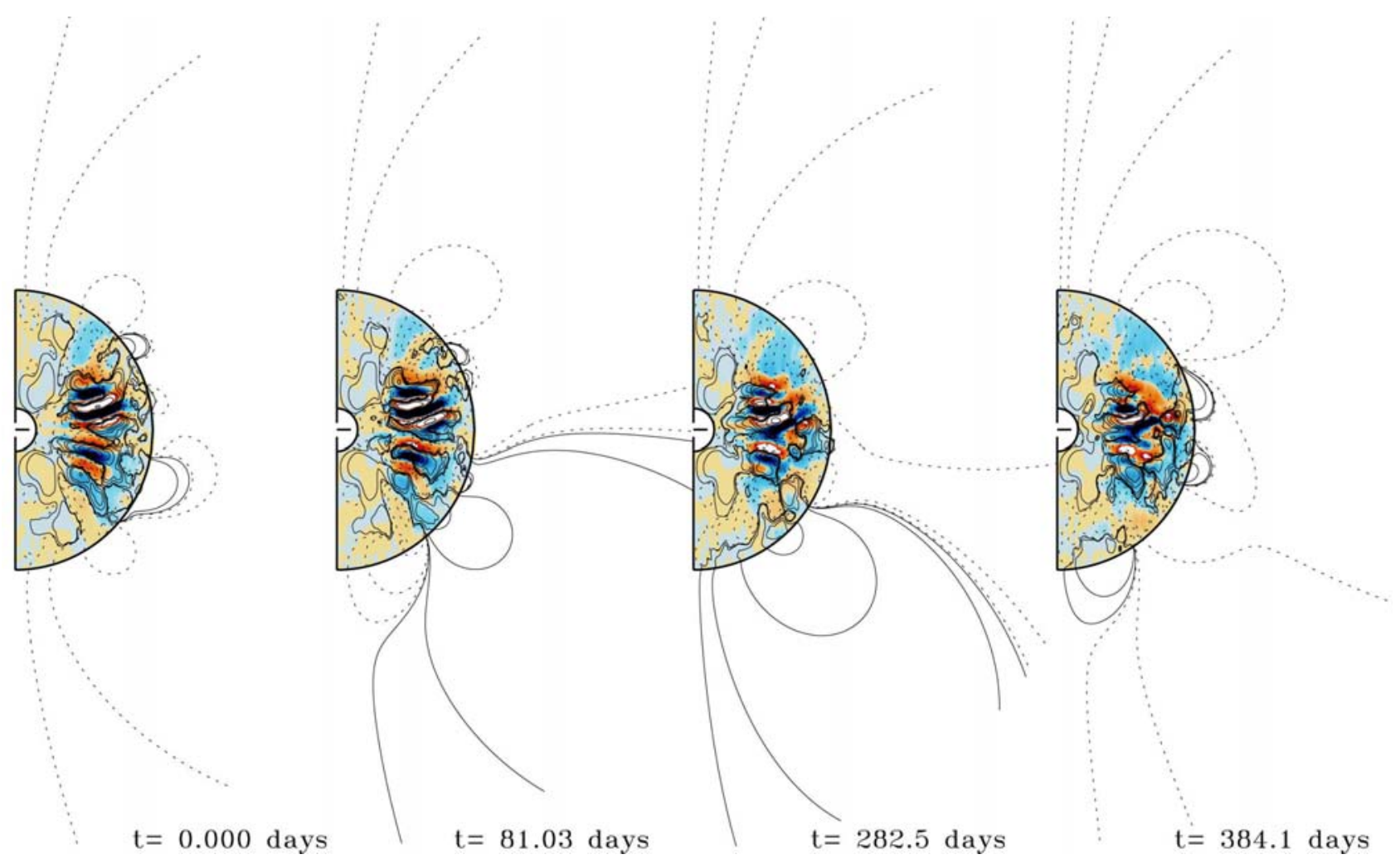

Fig. 8 (online colour at: www.an-journal.org) Evolution of the axisymmetric poloidal magnetic field after reaching the saturation state at $t=1300$ days. Contours of axisymmetric poloidal magnetic field are shown at different times with clockwise orientation (continuous line) and counterclosewise (dotted line) both within the star and outside it. The color scale between $-3000 \mathrm{G}$ (blue) and $3000 \mathrm{G}$ (red) shows the strength of the axisymmetric toroidal magnetic component within the star.

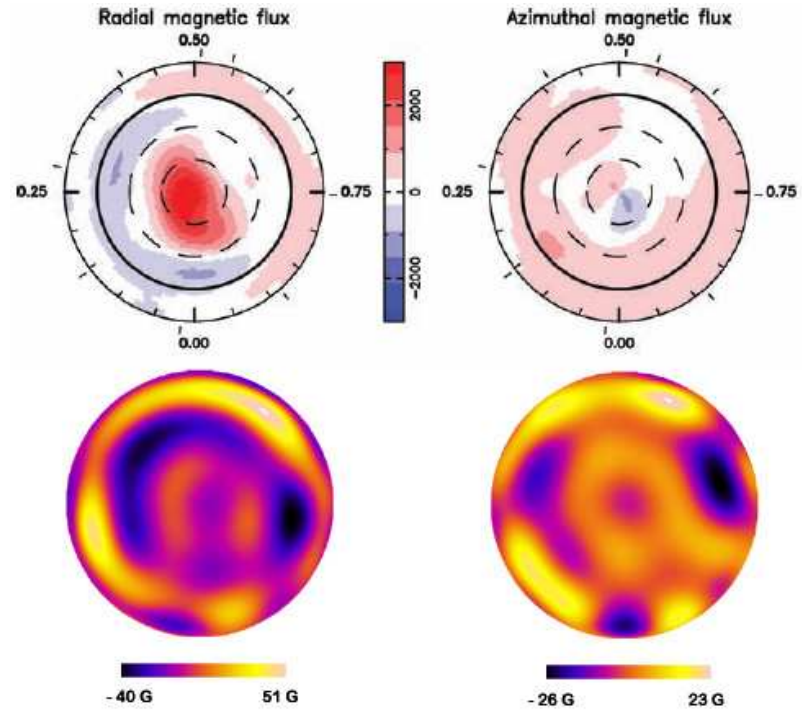

Fig. 9 (online colour at: www.an-journal.org) Magnetic field reconstruction of radial and azimuthal components from observational data of February 2006 (from Donati et al. 2008; top row) and from our dynamo computation (bottom row) after $t=1700$ days.

Otherwise, it is also possible that we are in a weak dynamo regime as Simitev \& Busse (2009) show for the regime of parameters we have, i.e. $\mathrm{Pr}=1$ and $\mathrm{Pr} / \mathrm{Pm}=1$ where it is expected that the dipolar component is much weaker than the small-scale field. We are currently exploring the sensitivity of our simulations of BP Tau to change of the parameters (mainly $\mathrm{Rm}$ and $\mathrm{Pm}$ ). We hope that we will be able to better characterize how such a potential strong mean field could be actually generated via fully $3 \mathrm{D}$ dynamo action in this nearly fully convective stage.

Acknowledgements. We want to thank the organizers for this very nice Thinkshop 7. The simulations were carried out on the national super computing centers such as the CCRT at CEA and JADE at CINES via GENCI project 1623. This work is part of the STARS ${ }^{2}$ project (http://www.stars2.eu) funded by a grant \#207430 of the European Research Council awarded to A.S.B.

\section{References}

Ballot, J., Brun, A.S., Turck-Chièze, S.: 2007, ApJ 669, 1190 Bessolaz, N., Brun, A.S.: 2011, ApJ 728, 115

Bessolaz, N., Zanni, C., Ferreira, J., Keppens, R., Bouvier, J.: 2008, A\&A 478, 155

Bouvier, J.: 2009, in: C. Neiner, J.-P. Zahn (eds.), Stellar Magnetism, EAS Publications Series 39, p. 199

Brown, B.P., Browning, M.K., Brun, A.S., Miesch, M.S., Toomre, J.: 2008, ApJ 689, 1354

Brown, B.P., Browning, M.K., Brun, A.S., Miesch, M.S., Toomre, J.: 2010, ApJ 711, 424

Brown, B.P., Miesch, M.S., Browning, M.K., Brun, A.S., Toomre, J.: 2011, ApJ 731, 69 
Browning, M.K.: 2008, ApJ 676, 1262

Brun, A.S., Miesch, M.S., Toomre, J.: 2004, ApJ 614, 1073

Donati, J.-F., Jardine, M.M., Gregory, S.G., et al.: 2007, MNRAS 380, 1297

Donati, J.-F., Jardine, M.M., Gregory, S.G., et al.: 2008, MNRAS 386, 1234

Donati, J.-F., Skelly, M.B., Bouvier, J., et al.: 2010, MNRAS 409, 1347
Glatzmaier, G.A., Gilman, P.A.: 1982, ApJ 256, 316

Goudard, L., Dormy, E.: 2008, AGU Fall Meeting Abstracts, A689 Iben, I., Jr.: 1965, ApJ 141, 993

Küker, M., Rüdiger, G.: 2005, AN 326, 265

Morel, P.: 1997, A\&A 124, 597

Morin, J., Donati, J.-F., Petit, P., et al.: 2008, MNRAS 390, 567

Simitev, R.D., Busse, F.H.: 2009, Europhysics Letters 851, 19001 